\title{
Gastrointestinal perforation in anti-NXP2 antibody-associated juvenile dermatomyositis: case reports and a review of the literature
}

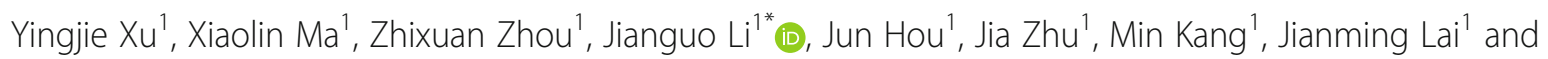
Xiaohui Li'

\begin{abstract}
Background: To summarize the characteristics of gastrointestinal (Gl) perforation in anti-nuclear matrix protein 2 (NXP2) antibody-associated juvenile dermatomyositis (JDM).

Methods: Five patients with Gl perforation from a JDM cohort of 120 cases are described. Relevant literature was reviewed.

Results: Five patients, including four females and one male, were included in the study. The age of onset of these patients ranged from 3.3 to 9.5 years with the median age of 5.0 years. When these patients were complicated by Gl perforation, childhood myositis assessment score (CMAS) ranged from 1 to 5 with the median score of 2. Myositis-specific antibody (MSA) spectrum analysis indicated that the five patients were anti-NXP2 antibody positive. The initial symptoms of Gl perforation were progressive abdominal pain and intermittent fever. Two patients also presented with ureteral calculus with hydronephrosis and ureteral stricture. Surgery was performed in four patients. One patient failed to undergo a repair as the perforation was high in position. For the other three patients, perforation repair was successful, of which two patients failed due to recurrent perforation. At 24 months postoperative follow-up, one patient was in complete remission on prednisone (Pred) and methotrexate (MTX) treatment, and her ureteral stricture had disappeared. The other four patients died. Adding these cases with 16 other patients described in the literature, the symptom at onset was progressive abdominal pain, which often occurred within 10 months after JDM was diagnosed. Perforation most commonly occurred in the duodenum, although it also occurred at multiple sites or was recurrent. The mortality rate of Gl perforation in JDM was 38\% (8/21).

Conclusions: All the five perforation cases in our study subjected to MSA analysis were anti-NXP2 antibody positive. The symptom at onset was abdominal pain. The most common site of perforation was the duodenum in the retroperitoneum, and the lack of acute abdominal manifestations prevented early diagnosis. Gl perforation may be a fatal complication in JDM, and early diagnosis is very important. More research is needed to determine the pathogenesis and predictive factors of GI perforation in JDM.
\end{abstract}

Keywords: Juvenile dermatomyositis, Gastrointestinal perforation, Anti-NXP2 antibody

\footnotetext{
* Correspondence: jianguo_li6@hotmail.com

'Department of Rheumatology, Capital Institute of Pediatrics-Peking

University Teaching Hospital, 2 Yabao Road, Beijing, China

Full list of author information is available at the end of the article
}

C C The Author(s). 2021 Open Access This article is licensed under a Creative Commons Attribution 4.0 International License, which permits use, sharing, adaptation, distribution and reproduction in any medium or format, as long as you give appropriate credit to the original author(s) and the source, provide a link to the Creative Commons licence, and indicate if changes were made. The images or other third party material in this article are included in the article's Creative Commons licence, unless indicated otherwise in a credit line to the material. If material is not included in the article's Creative Commons licence and your intended use is not permitted by statutory regulation or exceeds the permitted use, you will need to obtain permission directly from the copyright holder. To view a copy of this licence, visit http://creativecommons.org/licenses/by/4.0/ The Creative Commons Public Domain Dedication waiver (http://creativecommons.org/publicdomain/zero/1.0/) applies to the data made available in this article, unless otherwise stated in a credit line to the data. 


\section{Background}

Juvenile dermatomyositis (JDM) is an autoimmune disease characterized by proximal myopathy and a characteristic rash. However, multiple systems, including the digestive system, can also be involved. The occurrence of gastrointestinal (GI) perforation is rare, but a high mortality rate has been reported due to atypical symptoms and difficulty in early diagnosis [1]. Myositisspecific autoantibodies (MSAs) are increasingly used to delineate distinct subgroups of JDM [2,3]. The presence of anti-nuclear matrix protein 2 (NXP2) autoantibodies substantially increases the risk of calcinosis across all ages and is associated with disease severity [4]. The role of MSAs in cases of JDM complicated by GI perforation has not been reported. Here, from among 120 patients with JDM, we report five cases complicated by GI perforation. All five patients had anti-NXP2 antibodyassociated JDM. In addition, we reviewed the other JDM cases with GI perforation reported in the literature to improve our recognition of this disease. Anti-NXP2 autoantibodies may facilitate early diagnosis of the disease.

\section{Methods}

We described five patients with GI perforation from a JDM cohort who were admitted to the Children's Hospital affiliated with the Capital Institute of Pediatrics, China, from January 2016 to December 2019. The inclusion criteria were: (1) age < 18 years old; (2) diagnosis of JDM based on the Bohan and Peter criteria for myositis [5]; and (3) GI perforation. Patients with other diseases that cause weakness or rash, or a clear alternative diagnosis were excluded from the study. Sixteen MSAs (MDA-5, NXP2, Jo-1, PM-SCL100, PL-7, PL-12, EJ, OJ, anti-Ro52, Ku, PM-Scl, SRP, SAE1, TIF1 $\gamma, \mathrm{Mi}-2 \alpha$ and Mi-2 $\beta$ ) were tested by immune dot blot hybridization using a commercial kit based on goat anti-human IgG antibodies (BlueDot Myositis 12 IgG, \#MYO12D-24, DTEK, Belgium). The study was approved by the Ethics Committee of Capital Institute of Pediatrics. We searched PubMed, Medline and Scopus using the keywords "juvenile dermatomyositis", "gastrointestinal perforation", and "perforation". These literatures with detailed cases which clearly diagnosed JDM and excluded Degos-like presentation with GI perforation were reviewed.

\section{Results (Table 1)}

\section{The general data of the 5 patients}

Five patients with GI perforation were identified from 120 cases in a JDM cohort (4.17\%). Four of these were females, with the age at onset ranging from 3.3 to 9.5 years (median age -5.0 years). All five patients showed severe rashes, skin ulcers, and weakness of the skeletal muscles (ranging from power $3 / 5$ to $1 / 5$ ). When these patients were complicated by GI perforation, childhood myositis assessment score (CMAS) ranged from 1 to 5 with the median score of 2, cutaneous assessment tool (CAT) activity score ranged from 2 to 7 with the median score of 6, CAT damage score ranged from 3 to 6 with the median score of 5 . No calcification was observed. Two patients also presented with ureteral calculus with hydronephrosis and ureteral stricture. Analysis of MSAs showed that the five patients were anti-NXP2 antibody positive. There are 31 patients with anti-NXP2 antibody positive in the cohort. The incidence of GI perforation in anti-NXP2 antibody positive patients in our study was $16.13 \%(5 / 31)$. One patient had increased level of cytomegalovirus (CMV)-DNA copies in blood. GI perforation occurred from 4 to 13 months after JDM was diagnosed. The symptoms at onset were severe abdominal pain, fever, GI bleeding and vomiting. Perforations occurred in the duodenum (3 patients), duodenum and colon (1 patient), and an unclear location (1 patient), and perforations at multiple sites or at recurrent times occurred in four patients. All patients were treated with corticosteroids and immunosuppressant agents [cyclophosphamide (CYC)/methotrexate (MTX)/cyclosporine A (CsA)]. One patient was treated with plasma exchange (PE) therapy, and two patients received rituximab (RTX). All five patients were given proton-pump inhibitors (omeprazole). Surgery was performed in four patients, of which one patient failed to undergo repair due to the high position of perforation. For the other three patients, perforation repair was successful, of which two patients failed due to recurrent perforation. At 24 months postoperative follow-up, one patient was in complete remission under Pred and MTX treatment, and her ureteral stricture had disappeared. The other four patients died.

\section{Case 1}

A 3.4-year-old girl, suffering from characteristic skin rash and muscle weakness was diagnosed with JDM in a local hospital and was treated with $\operatorname{Pred}(2 \mathrm{mg} / \mathrm{kg} / \mathrm{d})$ and MTX (12 mg/m²/wk). Muscle weakness improved after 3 months of treatment, following which the dosage of Pred was reduced. When Pred was reduced to $10 \mathrm{mg} / \mathrm{d}$, the girl developed infection in the lungs, skin ulcers around the anus and abdominal pain. One month later, a perforation in the transverse colon was confirmed, and colostomy was performed in a local hospital. She received Pred, CYC, intravenous immunoglobulin (IVIG) and MTX, after which her abdominal pain disappeared, and her muscle strength improved. Seven months after JDM diagnosis, ureteral calculus with hydronephrosis and ureteral stricture were diagnosed and treated via 


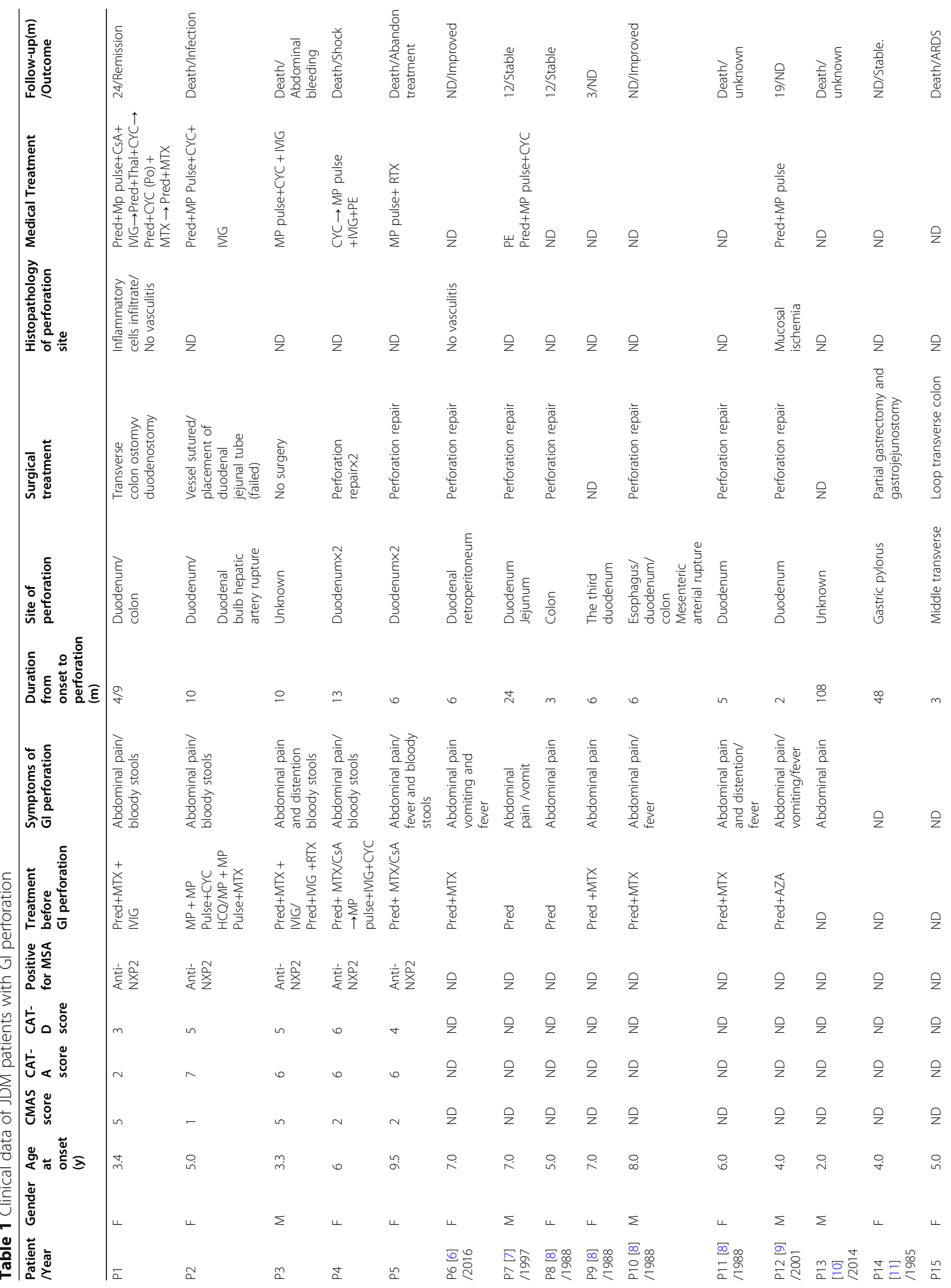




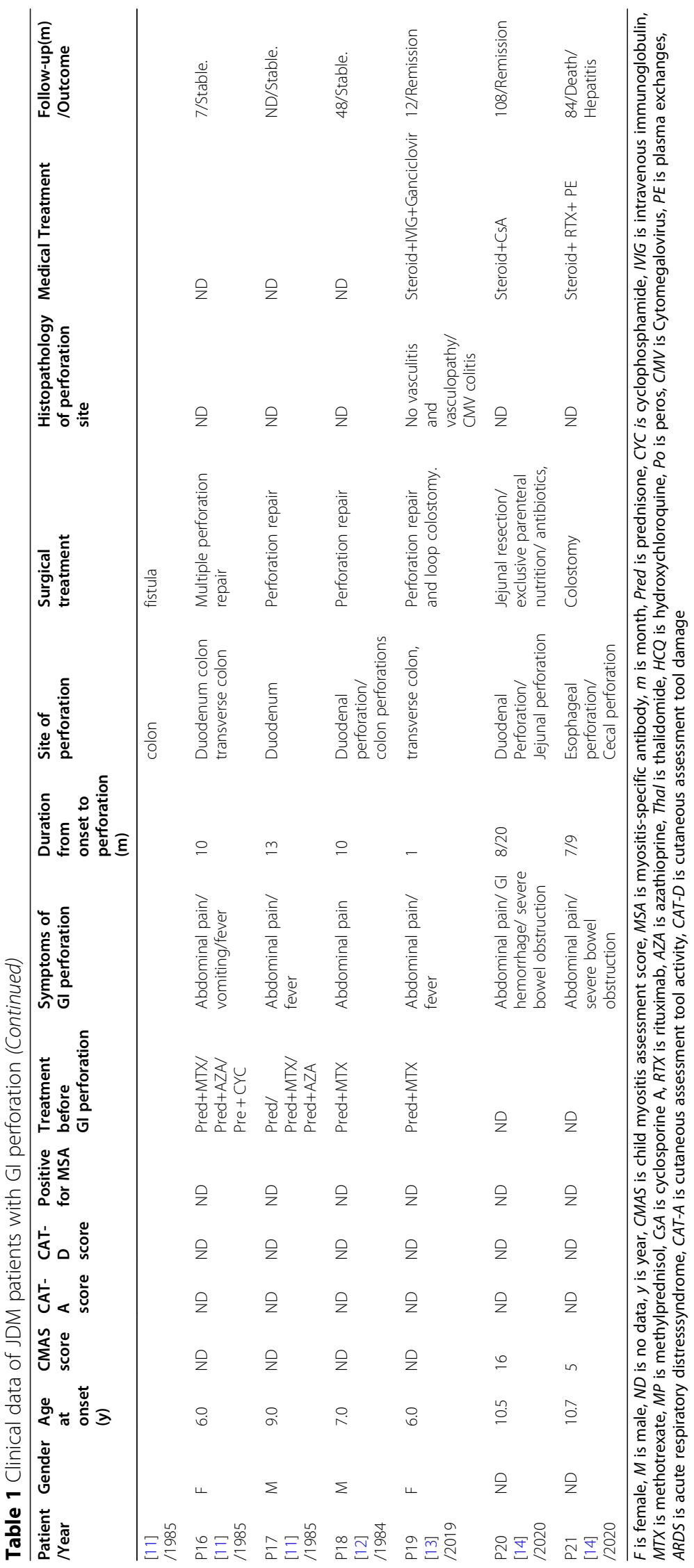


ureteroscopy. Nine months after JDM diagnosis, she developed severe abdominal pain and worsening muscle weakness, for which pulse methylprednisolone (MP) and CsA were given. Later, GI bleeding and fever recurred. She was transferred to our emergency department. She had shock, and her contrast-enhanced computed tomography (CT) abdomen indicated encapsulated effusion and pneumatosis, duodenal perforation and an abscess, which were confirmed during surgery. After duodenostomy, Pred, pulse CYC and thalidomide were given, and when her condition stabilized, we switched to oral Pred, CYC and MTX. At 24 months postoperative follow-up, she was in complete remission with Pred and MTX treatment, and her ureteral stricture had disappeared.

\section{Case 2}

A 5-year-old girl suffering from characteristic skin rash and muscle weakness was diagnosed with JDM in a local hospital and treated with Pred, MTX and hydroxychloroquine (HCQ), after which her muscle strength improved. But her parents stopped the treatment. Twelve months after JDM diagnosis, she was admitted to our emergency department due to abdominal pain, vomiting, and dark coloured stools. Physical examination showed a poor mental response, painful expression, severe malnutrition, High spring sign (+), partially scabbed skin ulcers in the lower jaw, popliteal fossa and axillae and muscle weakness. A contrast-enhanced CT abdomen indicated peritonitis, intestinal perforation and a right renalureteral calculus with pelvicalyceal-ureteral dilation. During surgery, multiple perforations of the duodenum and ruptured right hepatic artery were found. The ruptured blood vessel was sutured, but duodeno jejunal tube placement was unsuccessful. The patient subsequently died of infections and haemorrhagic shock.

\section{Case 3}

A 3.3-year-old boy suffering from characteristic skin rash and muscle weakness was diagnosed with JDM and treated with Pred, MTX and IVIG in a local hospital. His symptoms improved after treatment; the dosage of Pred was reduced, and MTX was stopped due to cataracts and abnormal liver function. The rash and muscle weakness worsened. Nine months after JDM diagnosis, physical examination revealed that severe proximal muscle weakness (power 3/5) and numerous purplish red rashes and ulcerative scabs which could be seen on the face and limbs. MP, RTX and IVIG were administered in our hospital. Two weeks later, he gradually developed abdominal pain, bloating, low back pain, right lower quadrant tenderness and rebound pain. Localized colitis was suspected based on a CT scan. His abdominal pain and distension were relieved after the administration of antibiotics, MP (20 mg/kg/d for 2 days) and IVIG.
Four days later, he developed fever, abdominal pain and distension, and an ultrasound showed echoic enhancement in the omentum of the right lower quadrant with weak peristalsis and ascites. MP, pulse CYC and IVIG were continued, and his temperature returned to normal with improved abdominal pain. However, 7 days later, he experienced severe abdominal pain and distension with a rapid decrease in his haemoglobin $(\mathrm{Hb})$ level (from $112 \mathrm{~g} / \mathrm{L}$ to $67 \mathrm{~g} / \mathrm{L}$ ). Ultrasound showed abdominal muscle inflammation with haemorrhage, and contrastenhanced CT showed generalized retroperitoneal infection with abdominal wall haemorrhage and intestinal perforation (Fig. 1). Surgical intervention was not possible in view of unstable vital signs, severe infection, and severe and extensive intestinal lesions that could not be surgically repaired. Repeated blood transfusions, antimicrobial agents, and treatment for the primary disease were given. He persisted to have abdominal pain and bloating. A slight change in body position caused massive abdominal wall bleeding. He died after returning home.

\section{Case 4}

A 7-year-old girl with a past history of JDM was treated 1 year earlier with MP and an immunosuppressant. She presented with progressive proximal muscle weakness, skin ulcers and intermittent abdominal pain for 1 month. Physical examination showed severe malnutrition, anemia, maculopapular rashes symmetrically distributed over the extensor sides of the joints of all extremities, partially scabbing skin ulcers on the extensor side of the left elbow and in both axilla, proximal muscle weakness and edema in both upper limbs. Her $\mathrm{Hb}$ level was $59 \mathrm{~g} / \mathrm{L}$. Abdominal contrast-enhanced CT did not indicate obvious abnormalities. Ultrasound of the abdomen suggested thickening of the abdominal bowel wall and poor bowel motility. After treatment with blood transfusion, MP and CYC were given. Her symptoms improved, and abdominal pain disappeared. $\mathrm{Hb}$ decreased again after 2 weeks, and fever and abdominal pain reappeared again after 4 weeks. Abdominal CT revealed bowel perforation. She immediately underwent surgical repair (Fig. 2). One week later, abdominal pain appeared again. Abdominal CT showed another intestinal perforation. Surgical repair and PE therapy were performed. Her abdominal pain was relieved, but she developed severe pulmonary infection. Three days later, she had manifestations of peritonitis and then developed seizures. Her blood pressure was normal, cerebrospinal fluid tests were negative, but her magnetic resonance imaging (MRI) brain showed that the right temporal lobe and bilateral parieto-occipital lobes were swollen with abnormal signals; patchy abnormal signals were observed in the right thalamus, showing long T2 signal, 


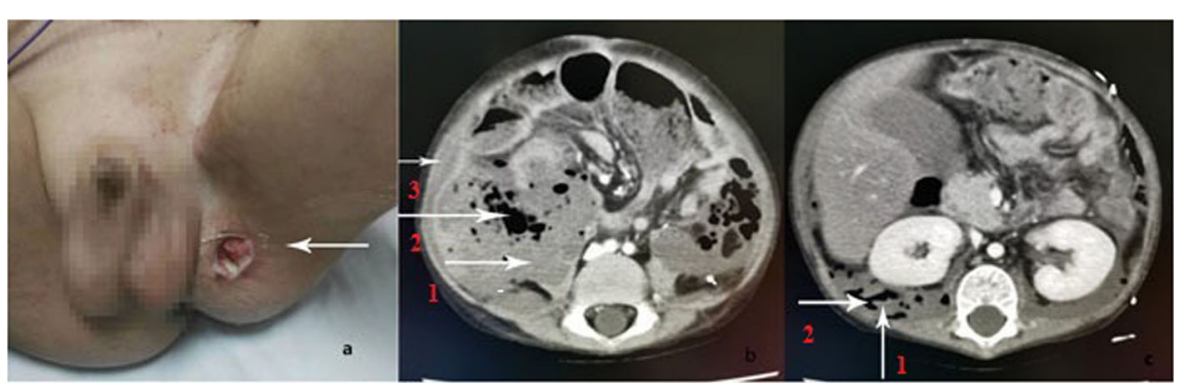

Fig. 1 a The thigh skin ulcers accompanied by swelling of the ipsilateral testis on Case 3(arrow) b. Contrast-enhanced CT showed retroperitoneal effusion (arrow 1), retroperitoneal gas (arrow 2) and abdominal wall hemorrhage (arrow 3) on Case 3. c Contrast-enhanced CT showed retroperitoneal effusion (arrow 1) and retroperitoneal gas (arrow 2) on Case 3

high T2 FLAIR signal, limited dispersion, and reduced $\mathrm{ADC}$ value. We speculated primary disease involving the nervous system. Seizures did not recur after treatment of the primary disease. A repeat MRI brain was not done. Pulse MP and anti-microbial agents were given. Surgical intervention was not possible. The patient died due to uncontrolled bleeding and refractory shock.

\section{Case 5}

A 10-year-old girl had been diagnosed with JDM 6 months earlier and received Pred and immunosuppressants. She presented with intermittent abdominal pain and fever for 1 month. She was initially treated with pulse MP and IVIG. But her muscle weakness and rash did not improve. She was admitted to our hospital with GI haemorrhage for 3 days. Physical examination showed a poor mental response, painful expression, severe malnutrition, anemia, scattered maculopapular rashes and ulcers distributed in the neck, axillae, buttocks, and extendible joints, and severe proximal muscle weakness. Her $\mathrm{Hb}$ level was $54 \mathrm{~g} / \mathrm{L}$. Ultrasound of the abdomen suggested thickening of the abdominal bowel wall and poor bowel movements. Pulse RTX and MP were given. Three days later, her abdominal pain worsened. Ultrasound of the abdomen showed intestinal perforation. During surgery, a $5-\mathrm{cm}$ perforation of the transverse part of the duodenum was observed, the perforation was repaired and a double cavity jejunal fistula was performed. Her abdominal pain was relieved. CMVDNA was detected in the blood, and she was given ganciclovir treatment. Four days later, she again experienced abdominal pain and muscle spasms. Considering the necrosis and perforation of the lower part of the duodenum, her parents withdrew treatment, and the patient died.

\section{Results of the literature review}

Sixteen patients of JDM with GI perforation were identified in PubMed, Medline and Scopus [6-18] which were published between 1984 and 2020. Eight patients were

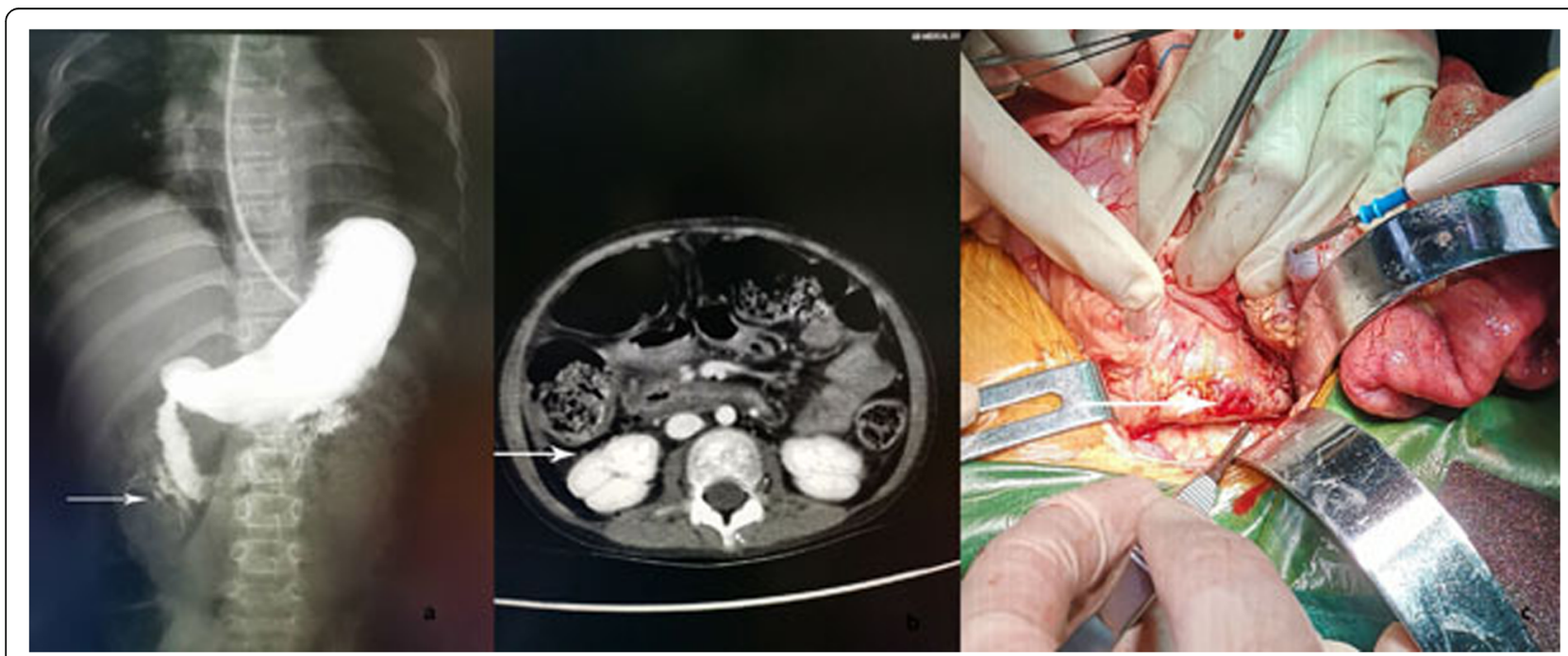

Fig.2 a Contrast leakage seen at the junction of the descending and horizontal segments of the duodenum on Case 4 (arrow). $\mathbf{b}$ Contrastenhanced CT showed retroperitoneal gas on Case 4 (arrow). c Duodenal perforation can be seen at surgery on Case 4 (arrow) 
female (50\%), six patients were male (37.5\%), and the sex of two patients was not recorded (12.5\%). The age of onset of these patients ranged from 2 to 10.5 years with the median age of 6.5 years. No clear MSA results were recorded in any case. Primary treatment with Pred and immunosuppressants did not have a significant effect. GI symptoms occurred from one to 108 months after treatment, most of which occurred within 10 months. The initial GI symptoms were severe abdominal pain with fever or vomiting. Perforations were reported in the duodenum $(10 / 16,62.5 \%)$, colon $(6 / 16,37.5 \%)$, jejunum (2/ $16,12.5 \%)$, oesophagus $(2 / 16,12.5 \%)$, gastric pylorus (1/ $16,6.25 \%)$, caecum $(1 / 16,6.25 \%)$ and an unclear location $(1 / 16,6.25 \%)$; perforations at multiple sites or recurrent perforations were reported in six patients $(6 / 16,37.5 \%)$. Only five patients had records regarding treatment of the initial disease, and they were treated with Pred or $\mathrm{MP}$ and an immunosuppressant (CYC/CsA). One patient underwent $\mathrm{PE}$ therapy, one patient received $\mathrm{PE}$ therapy with RTX and surgery was performed in 14 patients. In particular, one patient was treated with ganciclovir for CMV infection. Four patients died (25\%), and the others were discharged from the hospital after they experienced relief of their symptoms. The clinical data of all 16 patients taken from the literature and the five patients in our study is summarized in Table 1.

\section{Discussion}

JDM, the most common inflammatory myopathy of childhood, is a rare systemic autoimmune vasculopathy that is characterized by weakness in proximal muscles and pathognomonic skin rashes [19]. Multiple systems can be involved, but GI perforations in anti-NXP2 antibody-associated JDM are rarely reported. From among 120 patients with JDM, we report five anti-NXP2 antibody-associated JDM complicated by GI perforation. Most patients in our study were females, with a proportion that was significantly higher than that for the other patients reported in the literature. At the age of onset of these five patients ranged from 3.3 to 9.5 years with the median age of 5.0 years, and GI perforations appeared between 4 and 13 months post diagnosis, which was similar to the 16 cases reported in the literature. When our patients were complicated by GI perforation, CMAS were very low, CAT activity score indicated moderate activity, which suggest that GI perforations may be associated with disease severity and activity. The initial symptoms of JDM with GI perforation were severe abdominal pain, abdominal distention, vomiting, fever and bloody stools. Early identification of perforation was very difficult. With regard to patient (P) 1 and P2, the doctors did not recognize that GI perforation had occurred after persistent abdominal pain for more than 1 month. The perforation in P3 occurred during hospitalization in our hospital and manifested as severe abdominal pain and distension with decreased bowel sounds. Repeated abdominal ultrasounds, X-rays and contrast-enhanced CTs indicated the presence of a low-signal mass in the right lower quadrant with bowel distension, thinning of the intestinal wall and local colitis without pneumoperitoneum or subphrenic pneumatosis. Transitory relief of symptoms occurred after treatment, but severe abdominal pain quickly reappeared, and contrast-enhanced CT and ultrasound showed intestinal perforation, diffuse abdominal wall bleeding and infection. We did not know the precise time when the perforation occurred, suggesting that once abdominal pain appears, perforation should be strongly suspected, and imaging should be performed. Perforation should be considered when abdominal pain is not relieved by active treatment in patients with JDM, and it can be diagnosed early with contrast-enhanced CT scans [6]. We also suggest that repeated scanning and GI angiograms are essential, if the diagnosis cannot be confirmed during the first scan. In the 16 patients in the literature, perforation often occurred in the duodenum (partially in the retroperitoneal area in P6) and the colon. In addition, multi-site or recurrent perforations were also observed in some patients (P7,P10,P16, P18,P20,P21), similar to the results of our study. Furthermore, arterial rupture near the perforation site often occurs, as observed in P2, who had ruptured hepatic arteries, and in P10, who had a ruptured duodenal proximal mesenteric artery; therefore, haemorrhage may also be considered when an unexplained decrease in the $\mathrm{Hb}$ level occurs in JDM patients. In addition, several patients have been described in literature with Degos-like lesions linked to GI perforation [20, 21]. A similar deposit complex and pattern of vasculopathy has been found in patients suffering from Degos disease and JDM [22]. We should pay attention to the identification of Degos-like disease. Degos like skin lesions were initially pale red papules followed by enlarged lesions, showing a characteristic pitting center with a concave navel porcelain white center and a red halo around the periphery. After the rash subsided, there was a white superficial scar. The rash is more common on the trunk and extremities, less common on the palms and feet, and often occurs in batches, old and new. The appearance and location of the rash in five patients of our study were different from Degos-like disease. Further hisopathological identification may be performed if necessary.

In addition, two patients (P1, P2) had urinary system involvement in this group of cases, including ureteral stones, uretero-pelvic dilation and ureteral stenosis. With remission of the primary disease, ureteral stenosis disappeared in P1. This leads us to speculate that ureteral stenosis may be related to vasculopathy involving the urinary system. Morita et al. [23] reported a case of dermatomyositis who developed duodenal perforation as 
well as ureteral stenosis, with biopsy of stenotic ureter showing calcification with granulomatous tissue without any vasculitic lesion. Ruby Haviv et al. [24] reported a case of juvenile polymyositis (JPM) with right ureteral obstruction secondary to necrosis. The histopathology report of the resected ureter revealed moderate, acute, and chronic inflammatory infiltrates within the ureter wall, urothelial erosions, superficial granulation tissue, and degenerated smooth muscle fibres within the muscularis propria; some of which were infiltrated with lymphocytes and plasma cells, and multiple calcifications within the lamina propria. It also showed fragments of gross calcifications, similar calcifications in other parts of the ureter and obstructing calcifications within small blood vessels. Ruby Haviv et al. considered calcified ureteral necrosis to be a feature of visceral vasculopathy, related to both JDM and JPM. The mechanism is obscure, and the presence of calcifications within visceral tissues might suggest the pathogenesis of the typical calcinosis related to these diseases [24]. Considering that NXP-2 is a type prone to calcification, it was not clear whether ureteral calculi and ureteral stenosis in P1 and P2 were related to vasculopathy with calcification, which needs further studies of visceral calcinosis.

The underlying mechanisms of GI perforation in JDM are still obscure. Some researchers have reported glucocorticoids, non-steroidal anti inflammatory drugs and MTX to be risk factors for GI ulceration and perforation, especially at 1 month post-treatment or with an accumulated dose of steroids higher than $1 \mathrm{~g}$ [17]. In our study, Pred was reduced to a small dose before GI symptoms appeared in P1. After perforation was identified, pulse MP pulse and CYC were given, and the patient's condition improved; thus, the perforation was not attributed to drug factors. Schneider et al. [6] assumed that ulceration in the proximal region and bulb of the duodenum was related to medication, while perforation in the descending part was related to vasculitis, causing intestinal ischemia and necrosis due to thrombogenesis and vascular occlusion. Recent researchers $[16,17]$ reported that perforated sites showed chronic vasculopathy rather than acute vasculitis. This chronic vasculopathy is characterized by narrowing or complete occlusion of multiple small and medium arteries, subintimal foam cells, fibromyxoid neointimal expansion, and significant luminal compromise with infiltration of macrophages through the muscle layers into the intima. Histopathology at the perforation site was recorded in three patients $(\mathrm{P} 6, \mathrm{P} 12, \mathrm{P} 19)$ in the literature $[6,9,13]$, and no vasculitis was noted. P12 exhibited mucosal ischemia, and P19 exhibited vasculopathy and CMV colitis. No manifestation of vasculitis was observed in the histopathology of the fistula. Histopathology of perforation site in P1 of our study showed inflammatory cells infiltrate and no vasculitic lesion was observed in the tissue. The other three patients were unable to undergo histopathology due to erosion of the perforation site. Therefore, whether chronic non-inflammatory vasculopathy causes ischemia and perforation must be considered. Bhaskaran et al. [13] reported a case of intestinal perforation in a child with JDM caused by CMV colitis. CMV-DNA was also detected in the blood of P5. No relevant pathological tests were performed. The other four patients did not undergo blood CMV-DNA tests. The role of CMV colitis in perforation requires further study.

The 120 patients in the JDM cohort were all tested by MSAs analysis, all five patients with GI perforation had strong positivity for anti-NXP2 antibody (3+), and no GI perforation was noted in other MSA patients, which led us question whether anti-NXP2 antibody is associated with GI perforation. After reviewing 16 cases in the literature, Besnard et al. [13] reported that most patients with severe GI manifestations were positive for antiNXP2 antibody or anti-TIF-1Y antibody. Unfortunately, no specific antibody type was recorded in two patients with GI perforation in this study. The other patients described in the literature had not been tested for MSAs. In 2017, Tansley et al. [25] mentioned that 14 children who were positive for anti-NXP2 antibody had ulcers. Aouizerate et al. [26] reported that anti-NXP2 antibody had a positive association with GI involvement in JDM. The study evaluated the clinical, biological, and histological manifestations of 23 JDM patients and performed a multivariate analysis of 26 histopathological parameters. A case-control study can be performed to further uncover the relationship between anti-NXP2 antibody and GI perforation, which may further reveal the pathological mechanism of GI perforation. Based on our experience in this study, or even early in disease, clinicians should be highly alert to the possibility of GI perforation when abdominal pain occurs on anti-NXP2 antibodypositive patients with JDM.

The mortality rate among our patients was higher than that reported in the literature, which might be due to their severe condition and poor response to various therapeutic drugs, delayed surgery, surgical difficulties at the perforation site, economic reasons and the deficient number of cases. When a patient has intermittent abdominal pain and GI ulcers, the primary disease should be treated more actively to prevent GI perforation. Once GI perforation occurs, the primary disease is not well controlled, and the prognosis is poor. We need more research to choose appropriate treatment strategy for these diseases.

\section{Conclusion}

In this study, the clinical details of five cases of antiNXP2 antibody-associated JDM complicated by GI 
perforation were summarized for the first time. Based on the these cases combined with a literature review, we suggest that once JDM patients present with abdominal pain, especially anti-NXP2 antibody-positive patients, GI perforation should be considered. For timely diagnosis, dynamic imaging is important. GI perforation may be a fatal complication of JDM, more research is needed to determine the pathogenesis and predictive factors of GI perforation in JDM.

\author{
Abbreviations \\ JDM: Juvenile dermatomyositis; NXP2: Nuclear matrix protein 2; \\ Gl: Gastrointestinal; MSA: Myositis-specific antibody; CMAS: Child myositis \\ assessment score; Pred: Prednisone; CYC: Cyclophosphamide; \\ IVIG: Intravenous immunoglobulin; MTX: Methotrexate; \\ MP: Methylprednisolone; CSA: Cyclosporine A; RTX: Rituximab; \\ AZA: Azathioprine; Thal: Thalidomide; PE: Plasma exchanges; \\ CMV: Cytomegalovirus; CT: Computed tomography; \\ HCQ: Hydroxychloroquine; Hb: Haemoglobin; MRI: Magnetic resonance \\ imaging; JPM: Juvenile polymyositis; CAT: Cutaneous assessment tool; \\ P: Patient
}

\section{Acknowledgement}

We would like to thank the Department of General Surgery, the Department of Intensive Medicine and the Department of Radiology of the Children"s Hospital Affiliated to the Capital Institute of Pediatrics for their contributions to the treatment of patients in our study.

\section{Authors' contributions}

YX wrote the first draft of the manuscript, contributed to patient management and to the literature review. XM made many contributions in the process of writing and revising the manuscript. ZZ supervised patient management. JL critically revised the manuscript and supervised patient management. JH, JZ, MK and JL contributed to patient management. XL revised the manuscript. All the authors read and approved the final version of the manuscript.

\section{Funding}

This work was supported by Beijing Municipal Administration of Hospitals Clinical Medicine Development of Special Funding Support (code: XMLX201813) and Capital Funds for Health Improvement and Research (code:2020-2-2102).

\section{Availability of data and materials}

The datasets during and/or analyzed during the current study available from the corresponding author on reasonable request.

\section{Ethics approval and consent to participate}

Parental informed consent for publication was obtained. This study was approved by the Ethics Committee of Capital Institute of Pediatrics.

\section{Consent for publication}

Written informed consent was obtained from the patient's legal guardians for publication of this case report and any accompanying images.

\section{Competing interests}

The authors declare that they have no competing interests.

\section{Author details}

'Department of Rheumatology, Capital Institute of Pediatrics-Peking University Teaching Hospital, 2 Yabao Road, Beijing, China. ${ }^{2}$ Department of Cardiology, Capital Institute of Pediatrics-Peking University Teaching Hospital, 2 Yabao Road, Beijing, China.
Received: 19 June 2020 Accepted: 9 December 2020

Published online: 06 January 2021

\section{References}

1. Gitiaux C, De Antonio M, Aouizerate J, et al. Vasculopathy-related clinical and pathological features are associated with severe juvenile dermatomyositis. Rheumatol. 2016;55:470-9.

2. Pachman LM, Khojah AM. Advances in Juvenile Dermatomyositis: Myositis Specific Antibodies Aid in Understanding Disease Heterogeneity. J Pediatr. 2018;195:16-27.

3. Deakin CT, Yasin SA, Simou S, et al. Muscle Biopsy Findings in Combination With Myositis-Specific Autoantibodies Aid Prediction of Outcomes in Juvenile Dermatomyositis. Arthritis Rheumatol. 2016:68(11):2806-2816.

4. Tansley SL, et al. Calcinosis in juvenile dermatomyositis is influenced by both anti-NXP2 autoantibody status and age at disease onset. Rheumatol. 2014;53:2204-8

5. Brown VE, Pilkington CA, Feldman BM, et al. Network for juvenile Dermatomyositis, Paediatric rheumatology European society (PReS). An international consensus survey of the diagnostic criteria for juvenile dermatomyositis. Rheumatol. 2006:45:990-3.

6. Schneider KO, Braeuninger C, Bergmann F, et al. The importance of computed tomography for the diagnosis of duodenal perforation in a Paediatric patient with juvenile Dermatomyositis. Klin Padiatr. 2016;228:216-8.

7. See Y, Martin K, Rooney M, Woo P. Severe juvenile dermatomyositis complicated by pancreatitis. Br J Rheumatol. 1997;36(08):912-6.

8. Downey EC, Woolley MM, Hanson V. Required surgical therapy in the pediatric patient with dermatomyositis. Arch Surg. 1988;123(9):1117-20.

9. Wang IJ, Hsu WM, Shun CT, et al. Juvenile dermatomyositis complicated with vasculitis and duodenal perforation. J Formos Med Assoc. 2001;100(12): 844-846.

10. Singh $S$, Suri $D$, Aulakh $R$, et al. Mortality in children with juvenile dermatomyositis: two decades of experience from a single tertiary care Centre in North India. Clin Rheumatol. 2014;33(11):1675-9.

11. Schullinger JN, Jacobs JC, Berdon WE. Diagnosis and management of gastrointestinal perforations in childhood dermatomyositis with particular reference to perforations of the duodenum. J Pediatr Surg. 1985;20(5):521-4.

12. Thompson JW. Spontaneous perforation of the esophagus as a manifestation of dermatomyositis. Ann Otol Rhinol Laryngol. 1984;93(5):Pt 1 464-467.

13. Bhaskaran $\mathrm{H}$, Balan $\mathrm{S}$. Unusual cause for intestinal perforation in juvenile dermatomyositis. BMJ Case Rep. 2019;12:e229395.

14. Besnard C, Gitiaux C, Girard M, et al. Severe Abdominal Manifestations in Juvenile Dermatomyositis. J Pediatr Gastroenterol Nutr. 2020;70(2):247-51.

15. Ghayad E, Tohme A, Ingea H. Digestive manifestations of juvenile dermatomyositis. A case report and review of the literature. J Med Liban. 1993:41(4):240-3.

16. Mamyrova $G$, Kleiner $D$, James-Newton $L$, et al. Late-onset gastrointestinal pain in juvenile dermatomyositis as a manifestation of ischemic ulceration from chronic endarteropathy. Arthritis Rheum. 2007;57:881-4.

17. Miller LC, Michael AF, Kim Y. Childhood dermatomyositis. Clinical course and long-term follow-up. Clin Pediatr (Phila). 1987;26(11):561-6.

18. Singh $S$, Suri $D$, Aulakh $R$, et al. Mortality in children with juvenile dermatomyositis: two decades of experience from a single tertiary care Centre in North India. Clin Rheumatol. 2014;33:1675-9.

19. Feldman $B M$, Rider $L G$, Reed AM, et al. Juvenile dermatomyositis and other idiopathic inflammatory myopathies of childhood. Lancet. 2008;371:2201-12.

20. Yeung JT, Ma JK, Yung AW. Degos'syndrome complicated by bowel perforation: focus on radiological fndings. Hong Kong Med J. 2013;19(2): 174-7.

21. Vardeh H, Margo C, Rosen S. Gastrointestinal pathology associated with dermatomyositis: presentation of three cases and a general review. AJ Surg Pathol Rev Rep. 2010;21:293-300.

22. Louie CY, DiMaio MA, Charville GW, et al. Gastrointestinal tract vasculopathy: clinicopathology and description of a possible "new entity" with protean features. Am J Surg Pathol. 2018;42(7):866-76.

23. Morita Y, Sakuta T, Nagasu H, et al. Bilateral ureteral stenosis and duodenal perforation in a patient with dermatomyositis. Mod Rheumatol. 2007;179(1): 54-6.

24. Haviv R, Zehavi T, Pomeranz A, et al. Juvenile polymyositis associated with ureteral necrosis: a diagnostic and therapeutic dilemma-case report and review of the literature. Clin Rheumatol. 2019;38(7):1925-9. 
25. Tansley SL, Simou S, Shaddick G, et al. Autoantibodies in juvenile-onset myositis: their diagnostic value and associated clinical phenotype in a large UK cohort. J Autoimmun. 2017;84:55-64.

26. Aouizerate J, De Antonio M, Bader-Meunier B, et al. Muscle ischaemia associated with NXP2 autoantibodies: a severe subtype of juvenile dermatomyositis. Rheumatol. 2018;57(5):873-9.

\section{Publisher's Note}

Springer Nature remains neutral with regard to jurisdictional claims in published maps and institutional affiliations.

Ready to submit your research? Choose BMC and benefit from:

- fast, convenient online submission

- thorough peer review by experienced researchers in your field

- rapid publication on acceptance

- support for research data, including large and complex data types

- gold Open Access which fosters wider collaboration and increased citations

- maximum visibility for your research: over $100 \mathrm{M}$ website views per year

At BMC, research is always in progress.

Learn more biomedcentral.com/submissions 